\title{
Optimization of vibration energy localization in quasi-periodic structures
}

\author{
Mariem Hbaieb ${ }^{1,2, ~ *}$, Najib Kacem $^{1}$, Mohamed Amine Ben Souf ${ }^{2}$, Noureddine Bouhaddi ${ }^{1}$, Mohamed Haddar $^{2}$ \\ ${ }^{1}$ Univ. Bourgogne Franche-Comté, FEMTO-ST Institute, CNRS/UFC/ENSMM/UTBM, Department of Applied Mechanics, 25000 \\ Besançon, France \\ ${ }^{2}$ Research Laboratory of Mechanics Modeling and Production, National Engineering School of Sfax, Univ. Sfax, 3038 Sfax, Tunisia
}

\begin{abstract}
A mechanical periodic structure in presence of component perturbations can be a seat of a localization of vibration energy. In fact, it is well known that mistuned components have larger response levels than those of perfect components. This results in a localized energy, which can be tapped via harvesting devices. In this study, the dynamic behavior of a quasi-periodic system consisting in weakly connected linear oscillators is investigated. The main objective is to optimize the mistuning parameter, the coupling stiffness and the damping coefficient in order to functionalize the imperfection, which leads to the maximization of the localized vibration energy.
\end{abstract}

\section{Introduction}

The presence of irregularities in nominally periodic structures can inhibit the propagation of vibrations within the structure [1]. These small perturbations, defined as mistuning, generally increase the maximum amplitude of the forced response in a well-defined region of the quasiperiodic structure.

Depending on the magnitude of disorder and on the strength of internal coupling for the system, the irregularities may localize the vibration modes and confine the vibrational energy to a region close to the source. [1].

Modern structures are built slender, multi-span and many a times same basic substructure is repeated, for example turbine blades, multi span bridges and repetitive space structures [2]. In fact, in a real situation, there will always be a slight difference between the substructures that are supposed to be perfectly identical because of the geometry, properties of the materials, manufacturing tolerances or degradation in service.

As a result, mistuning may cause high vibration stresses and can have detrimental impact on the high cycle fatigue life of the component. [3].

In order to avoid the phenomena of localization of vibratory energy unexpected, several studies are oriented towards the optimization of the geometry of the nominally periodic structure for the reduction of the effects of disorder $[4,5]$.

Another approach seeks to amplify the localized vibratory energy in order to store it and use it in several applications such as energy harvesting [6, 7], energy trapping, vibration isolation...
In this paper, an oscillator system, represented for coupled cyclic structures, is chosen to study the combined effects of mismatch, stiffness coupling, and damping on the vibrational localization of cyclic structures.

In this study, the dynamic behavior of a quasi-periodic system consisting in weakly connected linear oscillators is investigated. The studied system involves eight oscillators coupled via linear springs while including two perturbations on two degrees of freedom (DOFs). The simplicity of the system makes it possible to effectively understand the characteristics of the vibration localization phenomenon involved in the cyclic structures.

The main objective of the present study is to optimize the parameters of the quasi-periodic structure in order to maximize the localized vibration energy.

The multi-objective optimization procedure consists in the maximization of the two mistuned DOFs response amplitudes using NSGA algorithm and Pareto front. Several numerical simulations have been performed to validate the proposed approach and to highlight the Pareto-optimal solutions, which can be used as a decisionmaking tool for the design of quasi-periodic structures with well mastered energy trapping.

\section{System Modeling}

Figure 1 shows the model for a N-oscillators system subjected to horizontal base excitation. Oscillators are connected to each other by spring of stiffness $k_{c}$ and each mass is grounded by springs of stiffness $\mathrm{k} . \mathrm{c}$ is assumed to be the proportional mechanical damping constant and $m_{n}$ is the mass of each oscillator.

* Corresponding author: mariem.hbaieb@enis.tn 
Mass of first oscillator is taken as nominal mass. Mistuning is introduced by varying the mass of two oscillators.

The development of equation of motion of a coupled oscillators is presented in this section.

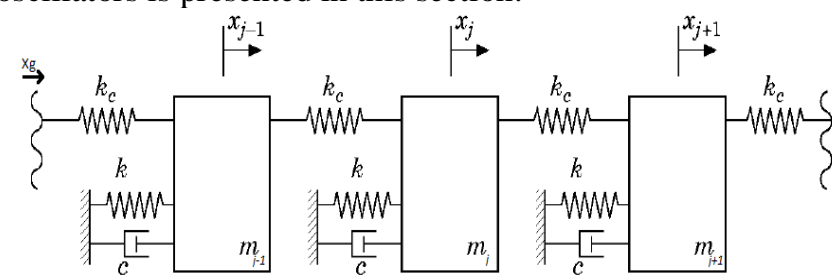

Fig. 1. A n-DOF spring-mass system Using the Lagrangien approach, the equations of motion can be written as follows.

$$
\left\{\begin{array}{c}
m_{n} \ddot{x}_{n}+c \dot{x}_{n}+\left(k+2 k_{c}\right) x_{n}-k_{c} x_{n-1}-k_{c} x_{n+1}=-m_{n} \ddot{x}_{g} \\
\text { with } n=1, . ., N
\end{array}\right.
$$

with represent a system of coupled linear equations, subjected to a basic excitation. We suppose that

$$
\begin{gathered}
\omega_{0}=\sqrt{\frac{k}{m}}, c=2 a m \omega_{0} \quad, \alpha_{n}=\frac{m_{n}}{m}, \beta=\frac{k_{c}}{k} \\
x_{n}=X_{n} e^{i \omega t} \quad \text { and } \quad x_{g}=X_{g} e^{i \omega t}
\end{gathered}
$$

where $\omega_{0}$ is the normal frequency of the sub-structure, $\alpha_{n}$ is the mistuning parameter, $\beta$ is the coupling stiffness and $a$ is the damping coefficient.

Then, we obtain the following equations:

$$
\left\{\begin{array}{c}
{\left[-\alpha_{n} \omega^{2}+2 \alpha_{n} a \omega_{0} \omega j+\omega_{0}^{2}(1+2 \beta)\right] X_{n}-\beta \omega_{0}^{2} X_{n-1}-\beta \omega_{0}^{2} X_{n+1}=\alpha_{n} \omega^{2} X_{g}} \\
\text { with } n=1, . ., N
\end{array}\right.
$$

The equations of motion can be written in the following matrix form :

$$
M \ddot{X}+C \dot{X}+K=M \ddot{X}_{g}
$$

The normal frequencies are such as the solutions of the following system:

$$
\left(-\omega^{2} M+K\right) X=0
$$

So

$$
\operatorname{det}\left(-\omega^{2} M+K\right)=0
$$

We may express all normal frequencies relative to the same reference frequency which is $\omega_{0}$, so that

$$
\omega_{n}=\omega_{0}(1+\varepsilon)^{\frac{1}{2}} \quad \text { with } n=1,2, . ., N
$$

where:

$$
\varepsilon=2 \beta\left(1-\cos \left(\frac{n \pi}{N+1}\right)\right) \text { with } n=1,2, \ldots, N
$$

The Taylor expansion of $\cos \left(\frac{n \pi}{N+1}\right)$ give the following approximate equation

$$
\omega_{i} \approx \omega_{0}\left(1+\frac{1}{2} \varepsilon\right)
$$

In the next section, the optimization of the localized vibration energy in the mistuned oscillator system will be investigated by a multi-objective approach.

\section{Multi-objective optimization problem}

For the rest of the paper, we will consider a system involves eight oscillators coupled via linear springs while including two perturbations on the $2^{\text {nd }}$ DOF and the $6^{\text {th }}$ DOF.

$$
\alpha_{2}=\frac{m_{2}}{m} \text { and } \alpha_{6}=\frac{m_{6}}{m}
$$

The problem of optimization of vibration energy localization in the quasi-periodic spring-mass system is formulated and solved as a combinatorial optimization problem.

The two mistuned DOFs response amplitudes are chosen as the objectives functions and the mistuning parameter, the coupling stiffness and the damping coefficient are the design variables.

The found approximations are used for solution of the problem by genetic optimization algorithms.

The optimization problem is posed as follows:

$$
\left\{\begin{array}{c}
\max _{\alpha_{2}, \alpha_{6}, \beta, a}\left(X_{2 \max }, X_{6 \max }\right) \\
X_{2 \max }\left(\alpha_{2}, \alpha_{6}, \beta, a\right) \\
X_{6 \max }\left(\alpha_{2}, \alpha_{6}, \beta, a\right) \\
\alpha_{2 \min }<\alpha_{2}<\alpha_{2 \max } \\
\alpha_{6 \min }<\alpha_{6}<\alpha_{6 \max } \\
\beta_{\min }<\beta<\beta_{\max } \\
a_{\min }<a<a_{\max }
\end{array}\right.
$$

(12)

In order to study the localization phenomenon, the vibration localization criterion is defined as the difference between the amplitude of displacement of the mistuned DOF and that of the two neighboring DOFs.

$$
\left\{\begin{array}{c}
\tau_{(k-1) / k}=\frac{\left|X_{(k-1) \max }-X_{k \max }\right|}{\max \left(X_{(k-1) \max }, X_{k \max }\right)} \\
\text { and } \\
\tau_{(k+1) / k}=\frac{\left|X_{(k+1) \max }-X_{k \max }\right|}{\max \left(X_{(k+1) \max }, X_{k \max }\right)}
\end{array}\right.
$$

In the next section, the optimal parameters of the structure will be determined by the multi-objective optimization method and Pareto front.

\section{Numerical results and discussion}

For the rest of the paper, we will consider the design parameters listed in the following Table 1.

Table 1. Design parameters for the corresponding periodic structure

\begin{tabular}{|c|c|c|}
\hline$m_{n}(k g)$ & $k(N . m)$ & $X_{g}(m)$ \\
\hline 0.05 & 100 & 0.001 \\
\hline
\end{tabular}


The intervals of variation of constraints of multi-objective problem are shown in Table 2.

Table 2. Intervals of variation of constraints of multiobjective problem

\begin{tabular}{|c|c|c|c|c|}
\cline { 2 - 5 } \multicolumn{1}{c|}{} & $\alpha_{2}$ & $\alpha_{6}$ & $\beta$ & $a$ \\
\hline$B_{\min }$ & 1 & 1 & 0.005 & 0.01 \\
\hline$B_{\max }$ & 1.1 & 1.1 & 0.015 & 0.02 \\
\hline
\end{tabular}

\subsection{Pareto front}

The main objective of Pareto front is to find a compromise between the two amplitudes displacement in order to maximize the sum of energy harvesting.

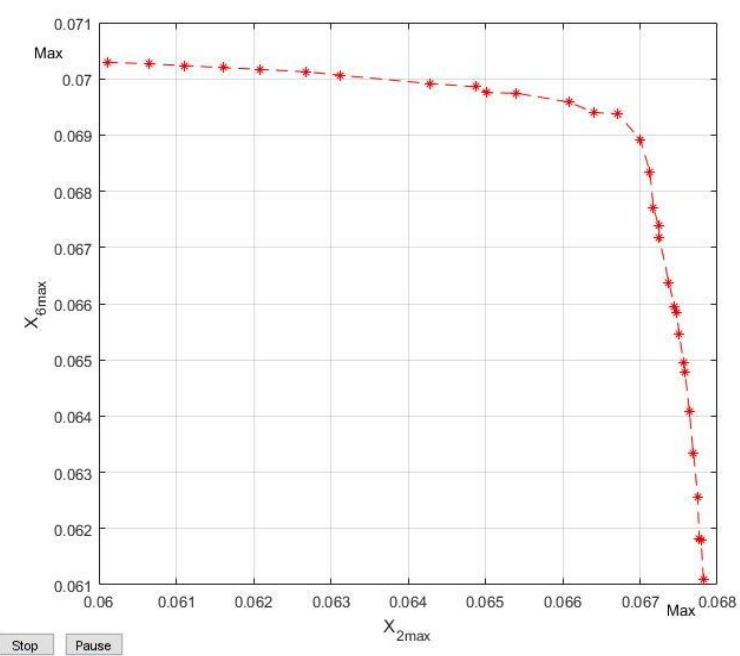

Fig. 2. Pareto front

We choose a point of the compromise zone:

$X_{2 \max }=66.8 \times 10^{-3} \mathrm{~m}$ and $X_{6 \max }=69.2 \times 10^{-3} \mathrm{~m}$ From this point, we look for the optimal values of the mistuning parameters $\alpha_{2}{ }^{*}$ and $\alpha_{6}{ }^{*}$, the coupling stiffness $\beta^{*}$ and the damping coefficient $a^{*}$.

We repeat the multi-objective study several times and from the presentation of the Pareto front we choose a point of the zone of compromise from which we determine the optimal parameters of the structure.

This approach yields the optimal intervals of the following structural parameters:

$$
\left\{\begin{array}{c}
1.031 \leq \alpha_{2}{ }^{*} \leq 1.041 \\
1.039 \leq \alpha_{6}{ }^{*} \leq 1.049 \\
0.014 \leq \beta^{*} \leq 0.015 \\
0.009 \leq a^{*} \leq 0.01
\end{array}\right.
$$

\subsection{Amplitude of displacement of mistuned DOFs}

Figures 3-a and 3-b are obtained for the coupling stiffness $\left(\beta^{*}=0.05\right)$ and the damping coefficient $\left(a^{*}=0.01\right)$ and present the variation of the amplitude of displacement of mistuned DOFs as a function of the mistuning parameters $\left(\alpha_{2}\right.$ and $\left.\alpha_{6}\right)$.

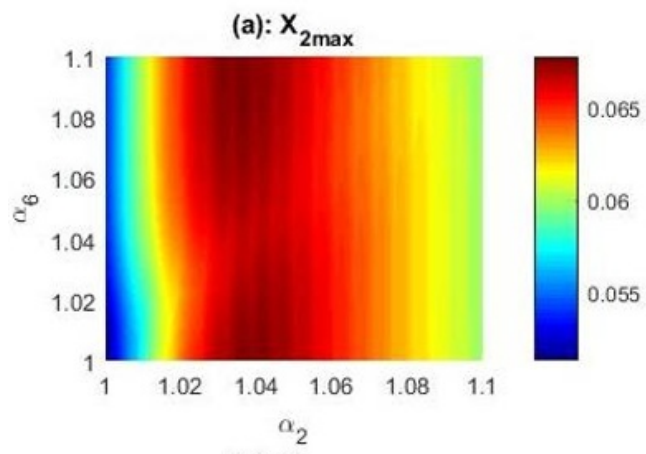

(b): $x_{6 \max }$

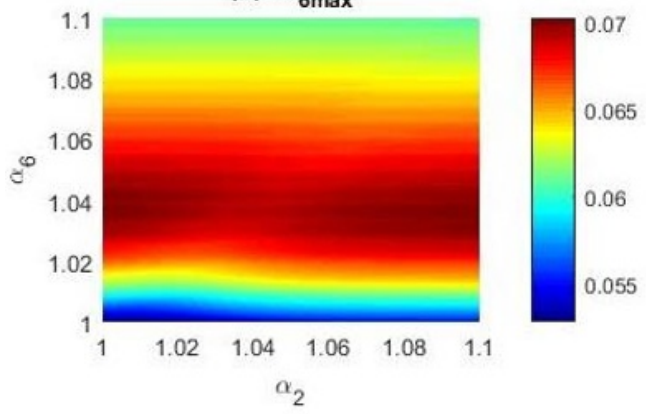

Fig. 3. Amplitude of displacement of mistuned DOFs according to mistuning parameters

Figure 3-a and 3-b show that around the optimal values of the mistuning parameters $\alpha_{2}$ and $\alpha_{6}$, the amplitude of displacement of the two mistuned DOFs is maximum.

\subsection{Frequency response}

For the two optimal mistuning parameters $\left(\alpha_{2}{ }^{*}=1.036\right.$ and $\left.\alpha_{6}{ }^{*}=1.044\right)$, the coupling stiffness $\left(\beta^{*}=0.05\right)$ and the damping coefficient $\left(\mathrm{a}^{*}=0.01\right)$, the frequency response of the system is shown in Figure 5

(a)

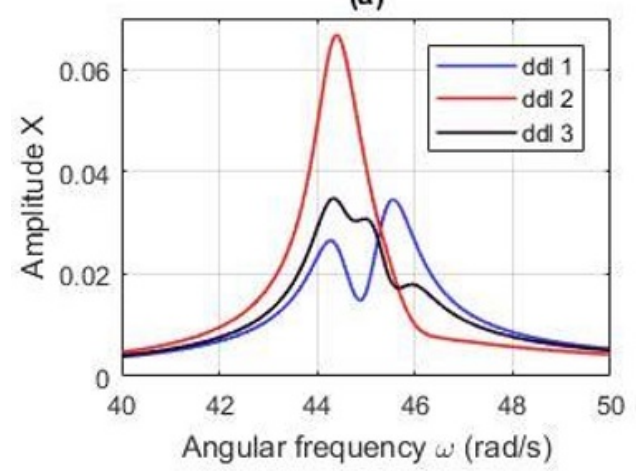

(b)

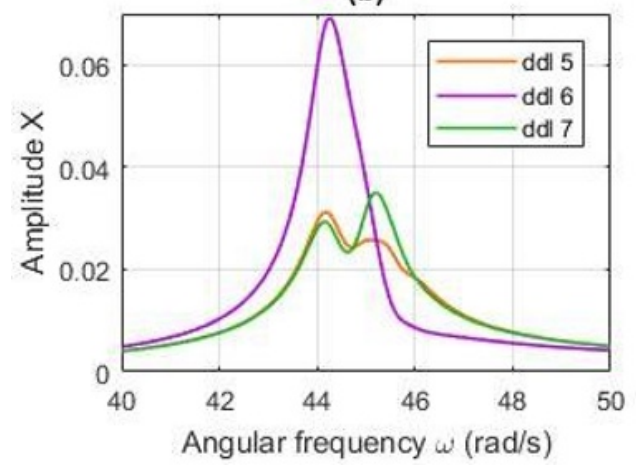

Fig. 4. Frequency response of the mistuned DOFs and of their two neighbors. 
Figures 4-a and 4-b are obtained for the mistuning parameters $\left(\alpha_{2}{ }^{*}=1.036\right.$ and $\left.\alpha_{6}{ }^{*}=1.044\right)$, the coupling stiffness $\left(\beta^{*}=0.05\right)$ and the damping coefficient $\left(a^{*}=0.01\right)$ showing the frequency responses of the mistuned DOFs and of their two neighbors.

These figures show that even two small mass perturbations at two DOFs of the periodic structure can lead to large difference in maximum amplitudes of oscillators.

It is also noted that the vibration energy will be located at the level of the mistuned mass with regard to a mass disturbance by adding matter ( $\alpha$ is greater than 1 ).

This localized energy can therefore be harvested from harvesting devices placed at the level of the $2^{\text {nd }}$ and the $6^{\text {th }}$ oscillators.

The vibration localization criterion of the two DOFs mistuned and their neighboring DOFs is defined as:

$$
\left\{\begin{array}{lll}
\tau_{2 / 1}=48.22 \% & \text { and } & \tau_{2 / 3}=54.87 \% \\
\tau_{6 / 5}=47.89 \% & \text { and } & \tau_{6 / 7}=49.35 \%
\end{array}\right.
$$

\section{Conclusions}

A generalized model of height horizontal oscillators coupled via linear springs while including two perturbations on two DOFs is developed. The objective of this study is to optimize the parameters of the quasiperiodic structure in order to maximize the localized vibration energy.

The problem of optimization of a quasi-periodic system mistuned is formulated and solved as a combinatorial optimization problem. An optimization method based on genetic optimization algorithm and Pareto front were developed for solving this problem.

In this paper, the parameters of a quasi-periodic system of coupled masses have been optimized by maximizing the two amplitudes of displacement of mistuned DOFs.

From the optimal parameters found, we verified the localization of the vibratory energy at the levels of the mistuned DOFs by the frequency response.

the periodic structures can be of very high dimension and which contains localized or distributed non-linearities.

The paper opens just what is happening in a small network. There is much more method of resolution when the network becomes narrower or in the presence of nonlinearity.

\section{References}

1. C. Pierre and E. H. Dowell, Localization of vibrations by structural irregularity, J. Sound Vib, 114, 19875 49-564

2. P. Malaji, S. Ali, Energy harvesting from near periodic structures, J. Vibration Engineering and Technology of Machinery, 23, 411-420 (2015)

3. J. Hou and Ch. Cross, Minimizing Blade Dynamic Response in a Bladed Disk Through Design Optimization, AIAA Journal, 43, 406-412 (2005)

4. E. Petrov, R. Vitali, and R. Haftka, Optimization of mistuned bladed discs using gradient-based response surface approximations, 41st Structures, Structural Dynamics, and Materials Conference and Exhibit, Structures, Structural Dynamics, and Materials and Co-located Conferences

5. Hong Hee Yoo, Jae Young Kim, Daniel J. Inman, Vibration localization of simplified mistuned cyclic structures undertaking external harmonic force, Journal of Sound and Vibration, 261, 859-870 (2003)

6. P. Malaji, S. Ali, Analysis of energy harvesting from multiple pendulums with and without mechanical coupling, The European Physical Journal Special Topics, 224, 2823-2838 (2015)

7. G. Litak, M. Friswell, C. Kwuimy, S. Adhikari, and M. Borowiec, Energy harvesting by two magnetopiezoelastic oscillators with mistuning, theoretical \& applied mechanics letters, 2, 043009 (2012) 